\title{
Avaliação da qualidade da referenciação dos CSP para a consulta de memória do hospital de Braga: um estudo descritivo
}

Ana Margarida da Cunha Torres Vieira, ${ }^{1}$ Ana Patrícia Rosa, ${ }^{2}$ Sandra Oliveira, ${ }^{3}$ Ana Sofia Costa ${ }^{4}$ Sofia Rocha, ${ }^{4}$ Álvaro Machado

\begin{abstract}
RESUMO
Objetivos: Caracterizar a referenciação à consulta de memória pelos cuidados primários, nomeadamente os motivos de referenciação, o estudo diagnóstico realizado ao nível dos cuidados primários e o tempo decorrido até à consulta hospitalar.

Tipo de estudo: Estudo observacional, transversal, descritivo.

Local: Hospital de Braga.

População: Utentes referenciados pelos cuidados de saúde primários à consulta de memória do hospital de Braga, com primeira consulta entre maio de 2011 e dezembro de 2015.

Métodos: Estudo realizado entre fevereiro e junho de 2016. Foram consultadas as referenciações informáticas dos utentes selecionados. As variáveis analisadas foram género, idade, profissão, escolaridade, estado civil, antecedentes de risco, motivos de referenciação, presença de defeito cognitivo, estudo diagnóstico analítico e imagiológico prévio e tempo decorrido até à referenciação e à consulta hospitalar. Resultados: Foram identificadas 290 referenciações, maioritariamente de utentes do género feminino (69\%), idade média de 75 anos, reformados (81\%), com menos de quatro anos de escolaridade (51\%). Cinquenta e oito por cento dos casos foram referenciados por perda de memória. Sessenta e oito por cento das referenciações indicavam antecedentes pessoais de risco e $10 \%$ antecedentes familiares de demência, $17 \%$ mencionavam a avaliação breve do estado mental, 51\% indicavam realização prévia de tomografia computorizada cerebral e $32 \%$ de estudo analítico. A referenciação demorou mais de 24 meses (55\%) e a consulta hospitalar entre sete a 12 meses (55\%). O principal motivo de referenciação está de acordo com outros estudos. As referenciações revelaram-se incompletas relativamente aos antecedentes de risco, ao rastreio cognitivo e à investigação diagnóstica prévia. As referenciações e a resposta hospitalar são efetuadas tardiamente.

Conclusão: Este estudo revelou lacunas na informação das referenciações que devem ser aperfeiçoadas, de modo a melhorar a articulação dos cuidados de saúde. Estes dados sugerem que pode haver limitações à abordagem destes doentes nos cuidados primários.
\end{abstract}

Palavras-chave: Memória; Demência; Cuidados de saúde primários; Diagnóstico precoce.

\section{INTRODUÇÃO}

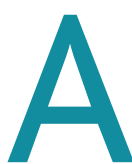
demência constitui uma expressão clínica de várias entidades patológicas que acarreta diminuição da qualidade de vida e elevada morbimortalidade. ${ }^{1}$ Implica um declínio em múltiplos domínios cognitivos, como da memória, atenção, linguagem, funções executivas, aprendizagem, capacidade percetiva motora ou cognição social, com impacto significativo nas atividades de vida diárias. ${ }^{2}$ Segundo a Organização Mun-

1. USF Manuel Rocha Peixoto

2. USF Alcaides Faria

3. USF do Minho

4. Hospital de Braga dial da Saúde, 47,5 milhões de pessoas têm demência a nível mundial, estimando-se um aumento para 75,6 milhões em 2030, com tendência a triplicar em $2050 .^{3}$ Um estudo recente identificou uma prevalência de demência em Portugal, no ano de 2013 , de 5,91\% na população acima dos 60 anos, aumentando gradualmente com a idade, sendo máxima no grupo etário com idade superior a 85 anos (24,8\%). ${ }^{1}$ Segundo a Direção-Geral da Saúde, em 2013 estavam registados $3,26 \%$ utentes com demência entre os utentes inscritos ativos nos cuidados de saúde primários (CSP) em Portugal Continental. ${ }^{4}$

No entanto, alguns estudos sugerem que a demência pode estar subdiagnosticada ou que existem obstáculos à 
abordagem da demência ao nível dos CSP. ${ }^{5}$ Estima-se que a maioria dos utentes com demência vigiados nos CSP é diagnosticada em estadios tardios (40-80\%). O maior desafio surge em distinguir precocemente as alterações cognitivas que fazem parte do processo de envelhecimento normal das alterações que surgem de processos degenerativos. ${ }^{6}$ Este atraso no diagnóstico conduz a uma maior taxa de hospitalizações evitáveis e a um recurso mais precoce às instituições de longa duração. ${ }^{5}$ Paralelamente, o diagnóstico precoce é especialmente importante numa altura em que se investe nas terapias modificadoras da doença, em que o tratamento possa influenciar o curso natural da doença. ${ }^{7}$ Além disso, permite ativar atempadamente os apoios da comunidade e reduzir a morbilidade psicológica sentida pelos cuidadores. ${ }^{8}$

Com este trabalho pretende-se conhecer as características da referenciação dos CSP à consulta de memória do serviço de neurologia do Hospital de Braga, a fim de se potenciar e melhorar a articulação entre diferentes níveis dos cuidados de saúde.

\section{MÉTODOS}

Realizou-se um estudo observacional, transversal e descritivo no serviço de neurologia do hospital de Braga. Selecionaram-se os utentes referenciados pelos CSP para a consulta de neurologia do hospital de Braga e triados para a consulta de memória, com primeira consulta entre maio de 2011 (desde que existem registos informáticos da consulta) e dezembro de 2015. Excluíram-se os utentes referenciados por outras especialidades do hospital de Braga, por outras consultas de neurologia, pelo serviço de urgência ou por outros hospitais da área de referenciação do serviço de neurologia.

Os dados foram recolhidos através dos registos da referenciação pelo médico de família (MF) à consulta de memória, acessíveis através do registo médico eletrónico utilizado no hospital de Braga. Incluíram-se dados sociodemográficos (idade, género, profissão, escolaridade, estado civil), os motivos de referenciação, a presença de defeito cognitivo aquando da referenciação pelos CSP através da avaliação cognitiva breve do estado mental (Mini Mental State Examination - MMSE), o estudo diagnóstico realizado previamente à referenciação (hemograma, glicemia, calcemia, ionograma sérico, função hepática, renal, tiroideia, vitamina B12, ácido fólico e teste sorológico para a sífilis e tomografia computorizada crânio-encefálica [TCCE], de acordo com a Norma de Orientação Clínica n. ${ }^{\circ}$ 053/2011, de 27/12/2011), ${ }^{9}$ a menção a antecedentes familiares em primeiro grau de demência, bem como a antecedentes pessoais de fatores de risco de demência (hi- pertensão arterial, diabetes mellitus, dislipidemia, doença cardiovascular estabelecida, abuso de álcool, depressão) ou a presença de sinais neurológicos focais. Recolheu-se ainda o tempo decorrido desde o início dos sintomas indicado na referenciação pelo MF e calculou-se o tempo decorrido entre a referenciação e a consulta hospitalar.

Elaborou-se uma base de dados informática devidamente codificada e analisaram-se os dados com recurso ao programa informático Microsoft Excel® e SPSS®) (Statistical Package for the Social Sciences), versão 21.

\section{RESULTADOS}

Identificou-se um total de 290 indivíduos referenciados pelos CSP para a consulta hospitalar de memória desde maio de 2011 até dezembro de 2015.

A maioria dos indivíduos referenciados eram do sexo feminino (69\%) e a idade variou entre os 37 e os 91 anos, com média de $75( \pm 9,73)$ anos. Em relação ao estado civil, $81 \%$ dos utentes eram reformados e $49 \%$ eram casados. Relativamente às habilitações literárias verificou-se que $23 \%$ dos utentes referenciados eram analfabetos e a maioria (51\%) apresentava entre um a quatro anos de escolaridade (Quadro I).

Analisando as variáveis indicadas nas referenciações pelos MF verificou-se que em $68 \%$ dos casos foi feita referência à presença/ausência de antecedentes pessoais de fatores de risco para demência, nomeadamente hipertensão arterial, diabetes mellitus, dislipidemia, doença cardiovascular estabelecida, abuso de álcool ou depressão (Quadro II). Em contraponto, em $90 \%$ dos casos não houve qualquer referência à presença/ausência de antecedentes familiares de demência, tal como não foi referido o valor do MMSE em $83 \%$ das referenciações. Das referenciações que apresentavam o valor do MMSE verificou-se que, em 54\% dos casos, o teste era positivo para defeito cognitivo de acordo com a escolaridade do doente, segundo os critérios definidos pelos pontos de corte nacionais de $1994 .{ }^{10}$

No que diz respeito aos motivos de referenciação averiguou-se que, em $58 \%$ dos casos, os utentes foram referenciados por perda de memória, $12 \%$ por alterações do comportamento/neuropsiquiátricas e $10 \%$ por deterioração cognitiva, entre outros (Quadro II).

Relativamente ao estudo diagnóstico efetuado pelos MF previamente à referenciação, constatou-se que $51 \%$ dos utentes realizaram TC-CE nos CSP. Adicionalmente apurou-se que apenas em $32 \%$ das referenciações havia referência à realização de estudo analítico prévio, sendo que $17 \%$ tinham análises pedidas, mas sem resultado, e apenas 6\% tinham estudo analítico completo (Quadro II).

Em relação ao tempo decorrido entre o início dos sin- 


\begin{tabular}{|c|c|c|}
\hline \multicolumn{3}{|c|}{$\begin{array}{l}\text { QUADRO I. Dados sociodemográficos dos utentes } \\
\text { referenciados dos cuidados de saúde primários para a } \\
\text { consulta de memória }\end{array}$} \\
\hline Total & \multicolumn{2}{|c|}{290 Indivíduos } \\
\hline Género & $\begin{array}{l}\text { Feminino } \\
\text { Masculino }\end{array}$ & $\begin{array}{r}201(69,3 \%) \\
89(30,7 \%)\end{array}$ \\
\hline Idade & \multicolumn{2}{|l|}{75,1 Anos $( \pm 9,7)$} \\
\hline $\begin{array}{l}\text { Estado civil } \\
(n=211)\end{array}$ & $\begin{array}{l}\text { Solteiro/a } \\
\text { União de facto } \\
\text { Casado/a } \\
\text { Divorciado/a } \\
\text { Viúvo/a }\end{array}$ & $\begin{array}{r}12(5,7 \%) \\
1(0,5 \%) \\
143(67,8 \%) \\
2(0,9 \%) \\
53(25,1 \%)\end{array}$ \\
\hline $\begin{array}{l}\text { Habilitações literárias } \\
(n=244)\end{array}$ & $\begin{array}{l}\text { Analfabeto } \\
\text { 1-4 Anos } \\
\text { 5-10 Anos } \\
\geq 11 \text { Anos } \\
\end{array}$ & $\begin{array}{r}67(27,5 \%) \\
147(60,2 \%) \\
17(7,0 \%) \\
13(5,3 \%)\end{array}$ \\
\hline $\begin{array}{l}\text { Situação face ao emprego } \\
(n=264)\end{array}$ & $\begin{array}{l}\text { Ativo } \\
\text { Desempregado } \\
\text { Reformado } \\
\text { Outro }\end{array}$ & $\begin{array}{r}15(5,7 \%) \\
7(2,6 \%) \\
236(89,4 \%) \\
6(2,3 \%)\end{array}$ \\
\hline
\end{tabular}

tomas e a referenciação à consulta hospitalar verificou-se que $47 \%$ dos indivíduos foram referenciados mais de 24 meses após o início dos sintomas, $14 \%$ entre os 12 e os 24 meses e $10 \%$ entre os 10 e os 12 meses (Figura 1a). A primeira consulta hospitalar de memória demorou entre sete a 12 meses a ser efetuada na maioria dos casos (55\%) e entre quatro a seis meses em $21 \%$ dos casos (Figura $1 \mathrm{~b}$ ).

\section{DISCUSSÃO}

A amostra deste estudo é composta maioritariamente por mulheres, o que pode ser explicado pela tendência demográfica observada no nosso país. De facto, em Portugal observa-se um aumento da esperança média de vida ao nascer, com consequente envelhecimento da população, sendo a esperança média de vida atual maior no sexo feminino. ${ }^{11}$ A proporção de indivíduos com 65 ou mais anos duplicou nas últimas décadas, atingindo os $19 \%$ nos censos de 2011, estimando-se que em 2060 a população idosa representará $36 \%$ a $43 \%$ da população portuguesa. ${ }^{1}$ Uma vez que a idade é um fator de risco para a doença de Alzheimer, a qual se estima que seja responsável por 50-70\% dos casos de demência, ${ }^{1}$ a idade média avançada (75 anos) da amostra deste estudo também é concordante com o esperado. A escolaridade baixa da amostra e o facto da maioria dos indivíduos estarem reformados são concordantes com a maior prevalência de indivíduos idosos no estudo, bem como com os dados do Censos 2011, que apontam para uma taxa de analfabetismo intermédia $(5,01 \%)$ na região Norte de Portugal, com a maioria da população residente com o primeiro ciclo de escolaridade completo (32\%). ${ }^{12}$

As recomendações internacionais preconizam um rastreio oportunístico nos indivíduos com risco moderado de demência (nomeadamente pela idade, história pessoal de risco cardiovascular elevado ou de depressão, antecedentes familiares de demência de Alzheimer ou presença de sintomas sugestivos de deterioração cognitiva), estudo atempado daqueles que apresentam queixas de memória e intervenção precoce nos CSP, que passa pela avaliação do défice cognitivo, estudo analítico e imagiológico (para a exclusão de causas reversíveis de demência), bem como informação ao doente, otimização da terapêutica e referenciação para os cuidados especializados. ${ }^{13-15}$

As orientações nacionais recomendam que os utentes com queixas de declínio cognitivo ou suspeita de demência sejam alvo de uma história clínica completa, adjuvada com informação de um familiar ou cuidador e que deve explorar as alterações nas atividades de vida diária, exame físico geral e exame neurológico. ${ }^{9}$ Recomenda-se que o défice cognitivo possa ser quantificado através de uma escala validada para a população em questão. ${ }^{9}$

Um estudo americano, publicado em $2014,{ }^{5}$ debruçou-se sobre a abordagem da demência ao nível dos CSP através da aplicação de um questionário a $1.500 \mathrm{MF}$ inscritos na Academia Americana. Dos respondentes, 56\% referiram que rastreavam indivíduos assintomáticos considerando a idade, história familiar e fatores de risco cardiovasculares, sendo os testes cognitivos mais aplicados o MMSE e o Teste do Relógio. Após um rastreio positivo, mais de $98 \%$ prosseguiam o estudo com análises e exames imagiológicos para excluir outras causas de demência. No presente estudo, o principal motivo de referenciação à consulta hospitalar foi a perda de memória. Este dado é concordante com outros estudos publicados que referem que as causas mais frequentes de referenciação para equipas mais especializadas são: declínio da memória, revisão da medicação, alterações do comportamento ou preocupação em relação à segurança. ${ }^{5}$

Nos doentes referenciados verificou-se que os fatores de risco para demência, nomeadamente os cardiovasculares, são referidos na maioria das referenciações pelos MF; no entanto, os antecedentes familiares de demência foram referidos numa minoria dos casos, sugerindo que os $\mathrm{MF}$ atribuem pouca relevância à história familiar para avaliação do risco e para diagnóstico diferencial na demência, o que contribui para uma menor precisão no rastreio e con- 
sequente subdiagnóstico.

Relativamente à quantificação do défice cognitivo, apenas $17 \%$ das referenciações indicavam o valor do MMSE e a escolaridade do doente, sendo o MMSE positivo em mais de metade dos casos. Isto indica que os MF recorrem pouco ao rastreio de défice cognitivo através do MMSE ou não consideram relevante a sua inclusão na referenciação. O MMSE é o teste mais amplamente usado a nível mundial e encontra-se validado para a população portuguesa. Apesar de conhecidas as suas limitações, os pontos de corte mais utilizados em Portugal são os que foram validados em 1994: 15 pontos para indivíduos analfabetos; 22 pontos para indivíduos com 1-11 anos de escolaridade e 27 pontos para indivíduos com mais de 11 anos de escolaridade. ${ }^{10}$ Ao longo dos anos surgiram novos estudos de validação destes pontos de corte. O último realizado na população portuguesa data de 2015 e considerou não apenas o nível de escolaridade, mas também a idade. ${ }^{16}$ Os autores atualizaram os pontos de corte para os seguintes valores: 26 pontos para indivíduos com idade superior ou igual a 50 anos e um a quatro anos de escolaridade; 27 pontos para indivíduos dos 25-49 anos com um a quatro anos de escolaridade; 28 pontos para indivíduos com cinco a nove anos de escolaridade, independentemente da idade; 28 pontos para indivíduos com idade superior, ou igual, a 65 anos e mais de cinco anos de escolaridade e 29 pontos para indivíduos com 25-49 anos e mais de 10 anos de escolaridade. ${ }^{16}$ Não obstante, estes dados são recentes e ainda pouco reconhecidos e utilizados, o que, aliado ao facto de o presente estudo ser retrospetivo, resultou no recurso aos pontos de corte mais antigos. consulta de memória

Não referido

Referido

Não referido

Referido

Não referido

- Perda de memória

- Outro

Não

Sim

Nenhum

Até ou igual a 4 itens

5-9 itens

Completo $\geq 10$ itens zada crânio-encefálica.
QUADRO II. Caracterização das variáveis em estudo na referenciação para a

\begin{tabular}{l|r} 
Estudo das referenciações & $\boldsymbol{n}(\%$ parciais $)$ \\
\hline AF de demência na referenciação & \\
Não referido & $262(90,3)$ \\
Referido & $28(9,7)$
\end{tabular}

AP de risco para demência

- Com fatores de risco para demência

- Sem fatores de risco para demência

MMSE na referenciação

- Valor positivo para demência de acordo com a escolaridade

- Valor negativo para demência de acordo com a escolaridade

$94(32,4)$

$196(67,6)$

$179(91,3)$

$17(8,7)$

Motivo de referenciação

- Alterações do comportamento ou neuropsiquiátricas

$240(82,8)$

$50(17,2)$

$27(54,0)$

$23(46,0)$

- Alteração no exame neurológico

- Deterioração funcional

- Deterioração cognitiva (funções executivas, linguagem, visuoespacial...)

- Refratariedade ao tratamento

- Dúvidas no diagnóstico

$169(58,3)$

$35(12,1)$

$0(0,0)$

$4(1,4)$

$28(9,7)$

$4(1,4)$

$12(4,1)$

$35(12,1)$

\section{Referenciação com TC prévia}

$143(49,3)$

Estudo analítico na referenciação

Pedido mas sem resultado

$147(50,7)$

Legenda: $\mathrm{AF}=$ antecedentes familiares; $\mathrm{AP}=$ antecedentes pessoais; $\mathrm{FR}=$ fatores de risco; itens = hemograma, glicemia, calcemia, ionograma sérico, função hepática, renal, tiroideia, vitamina B12, ácido fólico e teste sorológico para a sífilis; MMSE = avaliação breve do estado mental; TC-CE = tomografia computori-

De acordo com as orientações nacionais, perante a suspeita de demência devem ser solicitadas análises laboratoriais, incluindo hemograma, glicemia, calcemia, ionograma sérico, função hepática, renal, tiroideia, vitamina B12, ácido fólico e teste serológico para a sífilis. $\mathrm{O}$ doente deve ainda fazer um estudo de imagem estrutural, nomeadamente 


\section{Tempo decorrido entre o início dos sintomas, a referenciação e a consulta hospitalar (\% do total de doentes)}

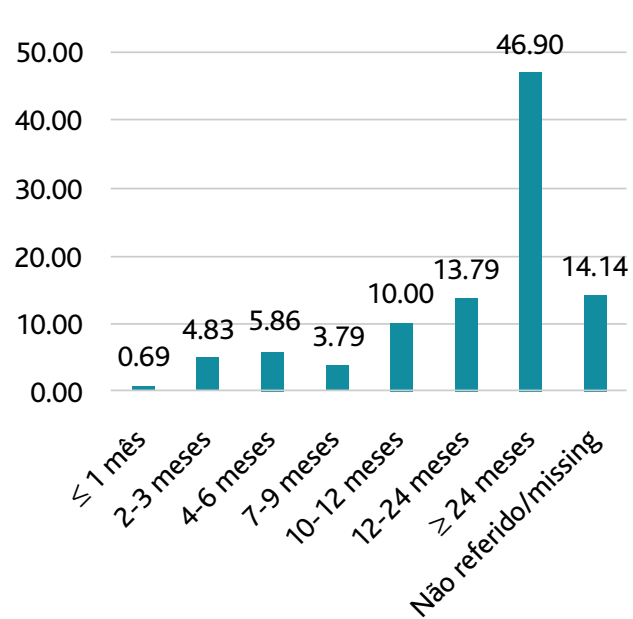

a) Intervalo de tempo decorrido entre o início dos sintomas e a referenciação pelos CSP

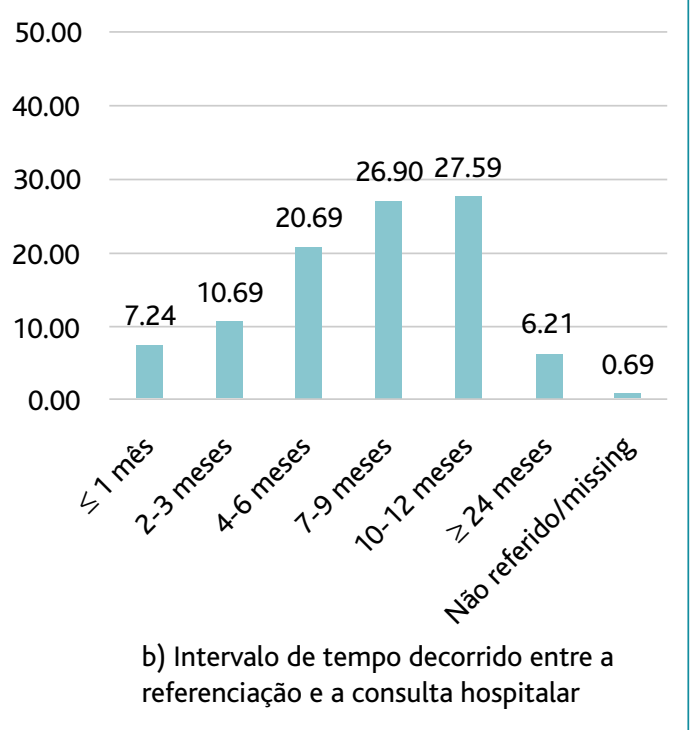

Figura 1. Resultados do tempo decorrido desde o início dos sintomas até à referenciação (a) e entre a referenciação e a consulta hospitalar (b).

uma TC-CE, no sentido de se excluírem causas tratáveis de declínio cognitivo e de fornecer informação para um diagnóstico etiológico específico. ${ }^{9} \mathrm{Na}$ amostra em questão cerca de metade dos utentes tinha informação sobre a realização de TC-CE nos CSP, mas a maioria não tinha indicação sobre a realização de análises laboratoriais, sendo que apenas $6 \%$ tinha o estudo analítico completo. Estes dados revelam uma deficiente investigação de causas tratáveis e potencialmente reversíveis nos doentes com suspeita de demência ao nível dos CSP previamente à referenciação. Não obstante, as queixas são valorizadas e, mesmo não se excluindo causas potencialmente tratáveis, os doentes são referenciados para a consulta hospitalar especializada, o que sugere que outras limitações poderão impedir a abordagem dos doentes com suspeita de demência pelos MF.

No contexto norte-americano, ${ }^{6}$ quando questionados sobre as principais barreiras ao diagnóstico e acompanhamento da demência, $78 \%$ dos MF referiram limitações de tempo nas consultas, $51 \%$ referiram que os testes cognitivos são demorados e $48 \%$ que existem limitações terapêuticas. De igual modo, a avaliação das expectativas e limitações sentidas pelos MF na avaliação e referenciação de doentes com suspeita de demência em contexto nacional parece ser um objetivo futuro importante para a melhoria dos cuidados e articulação interinstitucional.
Apesar de o rastreio de défice cognitivo ter demonstrado aumentar o número de casos identificados, o seu valor tem sido questionado, em parte devido à falta de dados que demonstrem melhor prognóstico, quer para os doentes quer para os cuidadores. Não obstante, sabe-se que apesar de o benefício da terapêutica na demência de Alzheimer, e doenças relacionadas, ser modesto, não modificar a história natural da demência, não ser curativo e de nem todos os doentes tolerarem a medicação disponível, as intervenções precoces são efetivas na melhoria da cognição, tratamento/prevenção da depressão e melhoria da qualidade de vida do doente e do cuidador. ${ }^{17}$ Além disso, a instituição precoce do tratamento farmacológico tem demonstrado atrasar a institucionalização destes indivíduos e a sua dependência. ${ }^{18}$ Relativamente às limitações terapêuticas, por exemplo, no nosso país não é possível nos CSP a prescrição de fármacos antidemenciais com o regime especial de comparticipação.

Este estudo indica que os MF estão altamente envolvidos na abordagem e acompanhamento dos doentes com suspeita ou diagnóstico de demência; no entanto, deparam-se com várias limitações na prática clínica. ${ }^{6}$ Efetivamente também na presente amostra os utentes foram referenciados vários meses após o início dos sintomas e esperaram, na maioria dos casos, entre sete e doze meses pela primeira consulta hospitalar, o que ultrapassa o tempo máximo de resposta garantida para a consulta desta especialidade. Algumas questões permanecem por responder relativamente às causas inerentes ao atraso no envio à consulta especializada. Seria importante perceber se este atraso se deve, por exemplo, à desvalorização da sintomatologia por parte dos utentes ou das famílias, quer por não 
reconhecerem os sintomas quer pelo estigma associado à demência, ou ainda por limitações inerentes ao próprio MF, como falta de conhecimento, incerteza diagnóstica, falta de tempo nas consultas ou a perceção de que o diagnóstico traz poucos benefícios em termos práticos para o doente. ${ }^{18}$ Além disso, seria importante também perceber se o atraso no envio à consulta especializada se reflete no prognóstico dos utentes. Um estudo que explorou as limitações que existem ao nível dos CSP em vários países Europeus e Norte Americanos concluiu que essas barreiras derivam, em parte, dos doentes e das suas famílias pelos motivos atrás expostos, mas sobretudo dos sistemas de saúde em que os clínicos trabalham (mais do que das suas competências), tendo sido apontadas limitações financeiras e de tempo nas consultas bem como a má articulação com os cuidados de saúde secundários (CSS). ${ }^{18}$

Há evidência de que a abordagem da demência por equipas multidisciplinares resulta em melhor cumprimento das recomendações, menor frequência das alterações do comportamento ou psicológicas, menor stress e depressão no cuidador, pelo que a abordagem multidisciplinar e atempada nestes casos é fundamental. ${ }^{5} \mathrm{O}$ diagnóstico precoce de demência, facilitado pelo rastreio, permite uma abordagem pró-ativa do doente e da família, permite planear os cuidados a longo prazo, prevenir a instalação de crises com necessidade de hospitalização e recurso ao serviço de urgência, bem como identificar aqueles que podem beneficiar de intervenções mais específicas, como sendo o treino do cuidador, o ensino de estratégias para lidar com o stress e participar em atividades saudáveis. ${ }^{5,19}$

Ao nível dos CSP seria interessante a promoção de parcerias estruturadas com organizações da comunidade para dotarem os doentes e as famílias de suporte educacional e apoio de equipas multidisciplinares (médicos, enfermeiros, psicólogos e assistentes sociais), bem como de protocolos que definam bem o papel dos CSP e CSS na abordagem da demência. Além disso, a implementação de medidas organizacionais que permitam ajustar o tempo das consultas nos CSP possibilitaria a aplicação das escalas de rastreio e o cumprimento das recomendações. Ao nível hospitalar, o alargamento do período de horário de consulta de memória e a referenciação direta para a consulta de memória, ao invés do que acontece atualmente em que a referenciação é feita para a consulta de neurologia e depois sujeita a triagem e marcação da consulta, poderia diminuir o tempo de espera da consulta especializada. Outras medidas passam pelo desenvolvimento de programas educacionais que encorajem os MF a uma prática clínica baseada nas recomendações nacionais. ${ }^{17}$
Algumas limitações importantes podem ser apontadas no presente estudo, nomeadamente o viés de recolha de dados registados apenas na referenciação e o viés de triagem dos utentes, uma vez que todas as referenciações passaram por um triador antes do envio à consulta de memória, o que poderá ter limitado a dimensão da amostra. Esta foi ainda limitada por ausência de registos informáticos anteriores a 2011.

Em conclusão, este estudo revelou lacunas na informação das referenciações dos utentes com suspeita de demência pelos CSP à consulta hospitalar, que devem ser aperfeiçoadas de modo a melhorar a articulação entre os diferentes níveis de cuidados e a qualidade dos cuidados de saúde prestados. É necessário fomentar o rastreio de défice cognitivo e a realização de estudo para exclusão de causas potencialmente reversíveis de demência ao nível dos CSP, bem como realizar esta avaliação em fase mais precoce da evolução da doença. Toda esta informação deve constar na referenciação do doente, de forma a melhorar a precisão da triagem e da prioridade na marcação da consulta. Para tal poderão contribuir sessões de formação multidisciplinar no hospital de Braga dirigidas aos MF da área de referenciação, com enfoque na abordagem da demência, estudo de primeira linha a realizar nos CSP e critérios de referenciação à consulta hospitalar. A qualificação da referenciação permitirá, em última análise, trazer benefício para o doente com suspeita de demência.

Este trabalho demonstrou resultados importantes, pioneiros em Portugal à luz do nosso conhecimento, sobre as referenciações à consulta de memória pelos CSP numa área geográfica significativa da região Norte do país. Espera-se que a divulgação do estudo sensibilize os profissionais de saúde para a temática e incentive a sua replicação noutros contextos a nível nacional, de modo a extrapolar e obter a sua validação externa. Além disso, são ainda necessários estudos que explorem eventuais limitações que os MF possam sentir na prática clínica que justifiquem estes resultados.

\section{REFERÊNCIAS BIBLIOGRÁFICAS}

1 Santana I, Farinha F, Freitas S, Rodrigues V, Carvalho A. Epidemiologia da demência e da doença de Alzheimer em Portugal: estimativas da prevalência e dos encargos financeiros com a medicação [The epidemiology of dementia and Alzheimer disease in Portugal: estimations of prevalence and treatment-costs]. Acta Med Port. 2015;28(2):182-8. Portuguese

2 American Psychiatric Association. Diagnostic and statistical manual of mental disorders. 5th ed. Washington: APA; 2013. ISBN 9780890425596

3 World Health Organization. Dementia [homepage]. Geneva: WHO; 2016 [updated $2017 \mathrm{Dec}$ ].Available from: http://www.who.int/mediacentre/factsheets/fs362/en/

4 Direção-Geral da Saúde. Saúde mental em números: programa nacional para a saúde mental, 2014. Lisboa: DGS; 2014. 
5 Parmar J, Dobbs B, McKay R, Kirwan C, Cooper T, Marin A, et al. Diagnosis and management of dementia in primary care: exploratory study. Can Fam Physician. 2014;60(5):457-65.

6 Stewart TV, Loskutova NL, Galliber JM, Warshaw GA, Coombs LJ, Staton EW, et al. Practice patterns, beliefs, and perceived barriers to care regarding dementia: a report from the American Academy of Family Physicians (AAFP) national research network. J Am Board Fam Med. 2014;27(2):275-83.

7 Panegyres PK, Berry R, Burchell J. Early dementia screening. Diagnostics (Basel). 2016;6(1):6.

8 Iliffe S, Mitchley S, Gould M, Haines A. Evaluation of the use of brief screening instruments for dementia, depression and problem drinking among elderly people in general practice. Br J Gen Pract. 1994;44(388):503-7.

9 Direção-Geral da Saúde. Abordagem terapêutica das alterações cognitivas: norma da DGS n. ${ }^{\circ}$ 53/2011, de 27/12/2011. Lisboa: DGS; 2011.

10 Guerreiro M, Silva AP, Botelho A, Leitão O, Castro-Caldas A, Garcia C, et al. Adaptação à população portuguesa da tradução do Mini Mental State Examination. Rev Port Neurol. 1994;1:9-10.

11 Instituto Nacional de Estatística. Censos 2016. Lisboa: INE; 2016.

12 Instituto Nacional de Estatística. Censos: resultados definitivos - Portugal, 2011. Lisboa: INE; 2012.

13 Prince M, Bryce R, Ferri C. World Alzheimer report 2011: the benefits of early diagnosis and intervention. London:Alzheimer's Disease International; 2011.

14 The Royal Australian College of General Practitioners. Guidelines for preventive activities in general practice. 9th ed. Melbourne: RACGP; 2012. ISBN 9780869064528

15 Cordell CB, Borson S, Boustani M, Chodosh J, Reuben D, Verghese J, et al. Alzheimer's Association recommendations for operationalizing the detection of cognitive impairment during Medicare Annual Wellness Visit in a pri- mary care setting. Alzheimers Dement. 2013;9(2):141-50

16 Freitas S, Simões MR, Alves L, Santana I. The relevance of sociodemographic and health variables on MMSE normative data. Appl Neuropsychol Adult. 2015;22(4):311-9.

17 Borson S, Frank L, Bayley PJ, Boustani M, Dean M, Lin PJ, et al. Improving dementia care: the role of screening and detection of cognitive impairment. Alzheimers Dement. 2013;9(2):151-9.

18 Koch T, lliffe S. Rapid appraisal of barriers to the diagnosis and management of patients with dementia in primary care: a systematic review. BMC Fam Pract. 2010;11:52.

19 Dubois B, Padovani A, Scheltens P, Rossi A, Dell'Agnello G. Timely diagnosis for Alzheimer's disease: a literature review on benefits and challenges. J Alzheimers Dis. 2016;49(3):617-31.

\section{CONFLITOS DE INTERESSE}

Os autores declaram não ter quaisquer conflitos de interesse.

\section{FONTES DE FINANCIAMENTO}

Os autores declaram não ter recebido subsídios ou bolsas para a elaboração do artigo.

\section{ENDEREÇO PARA CORRESPONDÊNCIA}

Ana Margarida da Cunha Torres Vieira

E-mail: anamctvieira@gmail.com

Recebido em 2017-02-25

Aceite para publicação em 2018-01-10

\section{ABSTRACT \\ EVALUATION OF THE QUALITY OF REFERRALS FROM PRIMARY CARE TO THE MEMORY CONSULTATION OF HOSPITAL DE BRAGA:A DESCRIPTIVE STUDY}

Objectives: To characterise the referrals from primary care to the memory, including the reasons for referral, the diagnostic study performed in primary care, and the time elapsed until the hospital consultation.

Type of study: Observational, cross-sectional, descriptive study.

Setting: Hospital de Braga.

Participants: Patients referred by primary care to the memory consultation of Hospital de Braga, with first consultation taking place between May 2011 and December 2015.

Methods: The study was carried out between February and June 2016. Informatic referrals of selected patients were retrieved. The variables analysed included gender, age, occupation, educational level, marital status, previous risk factors, reasons for referral, presence of cognitive impairment, previous diagnostic study, and time elapsed until referral and hospital consultation.

Results: We identified 290 referrals, mostly concerning female patients (69\%), mean age of 75 years $(69 \%)$, pensioners $(81 \%)$ and with less than 4 years of schooling $(51 \%)$. Fifty eight percent of the cases were referred by loss of memory. Sixty eight percent of the referrals had a personal history of risk, $10 \%$ has a family history of dementia, $17 \%$ had a brief assessment of mental status, $51 \%$ had a previous computed tomography scan, and $32 \%$ had previous analytic investigation. In $55 \%$ of the cases, the referral took more than 24 months and the waiting time until hospital consultation took between 7-12 months. The main reason for referral is in agreement with other studies. The referrals were incomplete regarding previous risk factors, cognitive screening and previous diagnostic investigation. Both referrals and hospital responses still take place late.

Conclusions: This study revealed information gaps in referrals that should be improved in order to optimise the transition between different levels of care. These results suggest that there may be limitations in approaching these patients in primary care.

Keywords: Memory; Dementia; Primary health care; Early diagnosis. 